Integration of Pharmacometrics and Pharmacoeconomics to Quantify the Value of Improved Forgiveness to Nonadherence: A Case Study of Novel Xanthine Oxidase Inhibitors for Gout
\end{abstract}

Hill McManus, Daniel; Marshall, Scott ; Soto, Elena; Hughes, Dyfrig

\title{
Clinical Pharmacology and Therapeutics
}

DOI:

10.1002/cpt.1454

Published: 01/09/2019

Cyswllt i'r cyhoeddiad / Link to publication

Dyfyniad o'r fersiwn a gyhoeddwyd / Citation for published version (APA):

Hill McManus, D., Marshall, S., Soto, E., \& Hughes, D. (2019). Integration of Pharmacometrics and Pharmacoeconomics to Quantify the Value of Improved Forgiveness to Nonadherence: A Case Study of Novel Xanthine Oxidase Inhibitors for Gout. Clinical Pharmacology and Therapeutics, 106(3), 652-660. https://doi.org/10.1002/cpt.1454

Hawliau Cyffredinol / General rights

Copyright and moral rights for the publications made accessible in the public portal are retained by the authors and/or other copyright owners and it is a condition of accessing publications that users recognise and abide by the legal requirements associated with these rights.

- Users may download and print one copy of any publication from the public portal for the purpose of private study or research.

- You may not further distribute the material or use it for any profit-making activity or commercial gain

- You may freely distribute the URL identifying the publication in the public portal ?

Take down policy

If you believe that this document breaches copyright please contact us providing details, and we will remove access to the work immediately and investigate your claim. 


\section{MANUSCRIPT TITLE}

Integration of pharmacometrics and pharmacoeconomics to quantify the value of improved forgiveness to non-adherence: A case study of novel xanthine oxidase inhibitors for gout

\section{AUTHORS}

Daniel Hill-McManus, BSc; Centre for Health Economics and Medicines Evaluation, Bangor University

Scott Marshall, PhD; Pharmacometrics, Pfizer Ltd, Sandwich

Elena Soto, PhD; Pharmacometrics, Pfizer Ltd, Sandwich.

Dyfrig Hughes, PhD; Centre for Health Economics and Medicines Evaluation, Bangor

University

\section{CORRESPONDING AUTHOR}

Dyfrig Hughes, Centre for Health Economics and Medicines Evaluation, Bangor Institute for Health and Medical Research, Ardudwy Building, Normal Site, Bangor University, Holyhead Road, Bangor, UK. LL57 2PZ. d.a.hughes@bangor.ac.uk. +44(0)1248 382950.

\section{CONFLICT OF INTEREST}

S.M. and E.S. are, or were, employees of Pfizer. D.H-M. and D.H. have no conflicts of interest to declare.

\section{FUNDING INFORMATION}

MRC Network of Hubs for Trial Methodological Research (HTMR), reference number MR/L004933/1- Q25, and the MRC North-West HTMR, reference number MR/K025635/1.

\section{KEYWORDS}

Pharmacometrics, pharmacoeconomics, value-based pricing, urate-lowering therapy, febuxostat, medication forgiveness 


\section{ABSTRACT}

Linked pharmacometric and pharmacoeconomic models provide a structured approach for assessing the value of candidate drugs in development. The aim of this study was to assess the utility of such an approach for identifying the properties of xanthine oxidase inhibitors (XOi) providing improved forgiveness to non-adherence and estimate the maximum reimbursement price. The pharmacometric and pharmacoeconomic models were used to simulate the time course of serum uric acid concentrations, and estimate quality-adjusted life years and costs for the XOi febuxostat and a range of hypothetical analogues. Compounds with reduced clearance or increased potency were more forgiving to missed doses, however, even following relatively large changes in these properties the predicted maximum reimbursement prices represented an increase of only $19 \%$ above febuxostat $80 \mathrm{mg}$. Linked pharmacometric and pharmacoeconomic modelling methods have the potential to inform early drug development by providing an indication of pricing options that may permit reimbursement. 


\section{INTRODUCTION}

Linked pharmacokinetic-pharmacodynamic-pharmacoeconomic (PKPDPE) models can provide a framework capable of testing the influence of drug pharmacology on long term clinical and economic outcomes, such as cost-effectiveness and value based pricing. ${ }^{1-4}$ This offers distinct advantages over conventional pharmacoeconomic analyses during clinical drug development by making explicit consideration of the relation between dose taking, dose-response, health outcomes and costs. Linked PKPDPE modelling can be used to predict the likelihood of therapeutic success and quantify the implications for pricing. One application, which exploits the mechanistic properties of this approach, is in determining the influence of non-adherence on the value of treatments. This represents a natural extension to previous research in which different patterns of adherence serve as inputs to $\mathrm{PK}^{5,6}$ and $\mathrm{PKPD}^{7-9}$ models, and provides a basis for estimating cost-effectiveness in preference to cost-efficacy. ${ }^{10}$

Imperfect medication adherence can limit the benefit of treatments, result in poorer outcomes for patients, and increase healthcare costs. ${ }^{11}$ Medication adherence can be decomposed into three distinct phases; 1) the initiation of treatment, 2) the degree to which a patient's dose taking matches the prescribed regimen while nominally adhering (implementation) and 3) the discontinuation of treatment (persistence). ${ }^{12}$ The design of medicines which remain effective when dose implementation is erratic - a property known as 'forgiveness' ${ }^{13,14}$ - may improve treatment effectiveness under conditions of routine care and provide added value. Conventional PK and PD modelling can offer insights on the impact of variable dosing on clinical endpoints ${ }^{15,16}$; however to our knowledge, there are no published methods for predicting the value of improving treatment forgiveness.

Despite the availability of effective urate-lowering therapies (ULTs) for gout, such as xanthine oxidase inhibitors (XOi) allopurinol and febuxostat, many patients fail to achieve serum uric acid target concentrations. Within clinical trials, allopurinol $300 \mathrm{mg} /$ day reduced serum uric acid (sUA) concentrations to below target $(6 \mathrm{mg} / \mathrm{dL})$ in $12 \%-41 \%$ of patients ${ }^{17-}$ 
${ }^{21}$; and febuxostat $80 \mathrm{mg} /$ day in $57 \%-76 \%$ of patients ${ }^{17-19}$. Rates of target attainment in routine practice are also low, and range from $22 \%$ (US primary care or rheumatology clinic) ${ }^{22}, 38 \%$ (UK primary care) ${ }^{23}$ to $45 \%$ (UK rheumatology $)^{24}$. A principal cause of treatment failure is non-adherence, with as few as $40 \%$ of patients being classed as adherent (medication possession ratio $>0.8$ ) using prescription claims data but with higher estimates obtained using other methods..$^{25,26}$

One potential way in which the next generation XOi could add value is through improved forgiveness. Of the many structurally dissimilar candidate lead compounds ${ }^{27}$, the potential for one to have such a property e.g. through reduced clearance or increased potency, could result in improved use-effectiveness. ${ }^{28}$ More forgiving drugs that retain greater effectiveness under real world adherence would be expected to result in quality of life benefits, and potentially impact on costs, compared with existing treatments. Many jurisdictions operate a form of value-based pricing where the maximum reimbursement price is linked to the added value of a medicine, in terms of both cost and health impacts. A higher maximum reimbursement price makes it more likely that a pharmaceutical company would achieve a return on investment.

This study uses real-world adherence data and PKPDPE modelling to simulate the effectiveness and determine the value of a series of hypothetical XOi. The aim was to assess the utility of using a PKPDPE model to link pharmacology to treatment effectiveness to the maximum reimbursement price in order to inform early decision making based on the predicted value that could be gained from developing a more forgiving drug.

\section{RESULTS}

The time courses of drug concentration in plasma and sUA concentration following single doses are presented in Figure 1 to illustrate the differences in pharmacokinetics and pharmacodynamics between febuxostat and hypothetical ULTs. The doses of hypothetical ULTs of group B (reduced $\mathrm{IC}_{50}$ and dose) and $\mathrm{C}$ (lower clearance and dose reduction) are 
reduced and consequently plasma concentrations of B4 and C4 are lower than febuxostat at $80 \mathrm{mg}$ and $120 \mathrm{mg}$. A4 (reduced $\mathrm{IC}_{50}$ ) results in the greatest reduction in sUA concentration but its effect is transient relative to $\mathrm{C} 4$ with an extended elimination half-life.

The predicted response rates for the hypothetical cohort over all PKPD model simulations are summarised in Table 1, where several possible measures of response have been presented. Febuxostat $80 \mathrm{mg}$ and $120 \mathrm{mg}$ were predicted to result in $55 \%$ and $64 \%$ of subjects with a mean sUA concentration below a $6 \mathrm{mg} / \mathrm{dL}$ target respectively. The hypothetical ULTs leading to the greatest proportion of responders was C4 (extended halflife with dose reduction) and A4 (increased potency without dose reduction) both achieving < $6 \mathrm{mg} / \mathrm{dL}$ in $75 \%$ of subjects. Scenarios assuming a greater potency and a reduced dose resulted in slightly lower response rates, down to $51 \%$, relative to febuxostat $80 \mathrm{mg}$.

Average response rates $(<6 \mathrm{mg} / \mathrm{dL})$ over all PKPD simulations by dose implementation groups, as shown in Table S1, are presented in Figure 2. There is very little differentiation between the ULTs when implementation is below $20 \%$ or above $90 \%$, with the best-worst treatment differences being between 0.8 and 10.3 percentage points. Greater differentiation occurs between $20 \%$ and $90 \%$, where the best-worst treatment difference ranges from 15.1 to 38.8 percentage points. A more pronounced pattern is observed for sustained treatment response, Figure S1, where there is no response predicted until at least $40 \%$ dose implementation. Only once implementation exceeds $70 \%$ of doses taken are high response rates (> 50\%) achieved.

Table 2 presents the estimated maximum reimbursement prices at which treatments are cost effective, based on differences in estimated lifetime QALYs and costs, resulting from expected changes in flare frequency. Prices are given using either febuxostat $80 \mathrm{mg}$ or 120 $\mathrm{mg}$ as the comparator. The highest maximum reimbursement prices are achieved by A4 and C4, which are expected to be cost effective at an annual price of $£ 376$, an increase of $19 \%$ on febuxostat $80 \mathrm{mg}$ at a threshold of $£ 20,000$ per QALY. 
Figure 3 shows the relationship between the responder rate and the pricing of a hypothetical ULT versus the comparator febuxostat $80 \mathrm{mg}$. The price axis is the difference between the maximum reimbursement prices at every response rate compared with the price of febuxostat $80 \mathrm{mg}$, hence the price at the response rate of $55 \%$ is fixed at $£ 0$. The two curves plot the relationship for a $£ 20,000$ per QALY cost effectiveness threshold and a probability of $10 \%$ and $50 \%$ of being cost effective at or below this threshold. This curve provides an estimate of the maximum reimbursement price for any response rate, and indicates that with $100 \%$ responder rate the maximum reimbursement price would be $£ 140$ above the annual cost of febuxostat $80 \mathrm{mg}$.

\section{DISCUSSION}

This study has demonstrated the application of linked PKPDPE models to inform drug development by estimating the maximum reimbursement price from drug pharmacology, using real-world data on medication adherence. In this case study, hypothetical XOi with reduced dose and extended duration of action were predicted to increase the proportion of treatment responders to a similar extent as those with increased potency alone. Simulations estimated a proportion of patients responding to treatment for these more 'forgiving' ULTs of between $60 \%$ and $75 \%$ compared with $55 \%$ for febuxostat $80 \mathrm{mg}$. Based on this improvement and assuming that treatment benefit is limited to a reduction in the frequency of acute gout flares, maximum reimbursement prices were estimated of between $4 \%$ and $19 \%$ above the $£ 317.72$ current annual cost of febuxostat using a cost effectiveness threshold of $£ 20,000$ per QALY gained.

The results of this study suggest that, under conditions of imperfect adherence, reduced clearance is of equal value as a target for early candidate selection as increased potency. The identification of compounds with long elimination half-lives, while maintaining bioavailability and potency may be challenging. However, some structurally diverse and highly potent XOi molecules have been identified and these may offer some potential lead candidates ${ }^{27}$, so further research and drug discovery endeavours could be justified. While 
there are alterative ULT mechanisms, such as uricosurics which lower sUA by stimulating its renal excretion, these have had limited success due to safety concerns. ${ }^{29}$ Similarly, administration of the enzyme uricase (e.g. pegloticase) that converts uric acid into the more soluble component, allantoin, is not widely used due to occurrence of anti-drug antibodies, injection site reactions and its high cost. ${ }^{30}$

The linkage of pharmacometrics with pharmacoeconomics remains relatively novel and there are few published examples, but has potential across a range of applications from early drug research and development ${ }^{2}$; in estimating cost effectiveness in alternative subgroups and treatment protocols ${ }^{31}$; in the evaluation of complex pharmaceutical interventions such as pharmacogenetic testing 32 ; and modelling health economics of treatments for use during pandemics ${ }^{33}$. Pharmacometrics has been used to study issues relating to medication adherence for some time (for example Vrijens et al. 2005 ${ }^{34}$ ). This study is the first, of which we are aware, to combine adherence, pharmacometrics and pharmacoeconomics to inform early drug design decisions. In doing so this further demonstrates the value of an interdisciplinary approach and the need to interconnect existing methods to improve efficiency in drug development. As such, linked PKPDPE modelling may be seen as an additional component within the model informed drug development paradigm. ${ }^{35}$

This study has advantages over conventional pharmacometric studies that do not assess the future value of compounds beyond market authorisation; and conversely, it has advantages over standard pharmacoeconomic practices which do not account for exposure response relationships. It has benefitted from a semi-mechanistic pharmacodynamic model that can account for the system dynamics resulting from intermittent dose taking. Unlike in some previous economic evaluations of ULTs ${ }^{36-38}$, in this study, the clinical benefits of lowering sUA concentration have been assumed to be limited to reduced frequency of flares alone. However, this is consistent with the findings of recent meta-analyses. ${ }^{39}$

Limitations of this study include the assumptions which were necessary in order to develop a model structure and to obtain parameters estimates. It has been assumed that the structure 
of the pharmacometric and pharmacoeconomic models provide a sufficiently accurate representation of ULTs and their impacts to make predictions. The PKPD model was developed from a variety of published sources without fully accounting for the additional uncertainty this introduced. Aspects of the pharmacoeconomic modelling, such as the frequency of acute gout flares, relied on survey data obtained from a small number of patients. We have not considered the safety aspects of hypothetical XOi which would inform dose selection, and would need to be accounted for in pharmacoeconomic models in terms of the cost and health implications. The adherence data was not collected in gout patients but does contain a wide variety of adherence patterns and the overall low level of adherence is consistent with studies on the routine use of ULTs.

Many jurisdictions make use of economic evaluations as a part of the decision making process of whether to reimburse medicines having obtained marketing authorisation. A new medicine failing to meet the criteria for cost effectiveness may not be marketable at a commercially viable price or gain sufficient market penetration for adequate return on investment. The framework used here provides a direct link between pharmacology and the probability of a medicine being cost-effective. These methods have the potential to inform early drug development by providing an indication of whether drug candidates possess the properties that would result in a maximum reimbursement price that justifies their progression through the long and costly drug development process.

\section{METHODS}

\section{Overview}

In the first stage, the time course of SUA was simulated based on real-world dose taking histories and using a range of drug models, representing both real-world and hypothetical XOi. This stage was repeated a large number of times with resampling from probability distributions for patient characteristics, including baseline sUA concentration, age and weight. In the second stage the post-treatment sUA was used to predict the annual 
frequency of acute gout flares over the patients' remaining lifetime and to estimate the overall costs and impacts on quality-adjusted life years.

\section{Pharmacometric and Pharmacoeconomic Models}

An existing two compartment pharmacokinetic (PK) model and multi-compartment semimechanistic pharmacodynamic (PD) model developed for febuxostat ${ }^{7}$ was used to simulate sUA concentrations. The structure of the pharmacodynamics model has been reproduced in Figure 4, while PK and PD model parameters are provided in the Supplementary Material. In addition to febuxostat at approved daily doses of $80 \mathrm{mg}$ and $120 \mathrm{mg}^{40}$, twelve 'hypothetical' ULTs were assessed by changing the values of potency or clearance parameters for febuxostat (Table 3).

The rationale for the clearance, potency and dose adjustments is that i) reduced clearance prolongs residual drug concentration (and therefore extends the duration of action), but for an unbiased comparison a dose reduction was made to maintain the same drug exposure (area under the concentration curve); and ii) for a given concentration, a more potent drug will result in greater effect and we have, therefore, tested scenarios with and without dose adjustments. In reality, decisions concerning dose would be guided by a consideration both of the efficacy and the safety profiles of a candidate compound. We have not considered safety in this study.

The pharmacoeconomic model used a Markov state-transition structure with a 3-month time cycle to estimate costs and QALYs in a cohort of patients eligible for ULT. An overview of the model structure is given in Figure S2, model parameters are provided in the Supplementary Material and a comprehensive description of the model can be found elsewhere. ${ }^{1}$ The approach to modelling cost effectiveness is consistent with the methods of the National Institute for Health and Care Excellence in the UK ${ }^{41}$, adopting the cost perspective of the National Health Service in the UK, a lifetime (50 year) time horizon, and 
costs and QALYs both discounted at a rate of 3.5\% per annum. ${ }^{42}$ The model was implemented in $\mathrm{R}$ version 3.5.1. ${ }^{43}$

For each ULT in Table 3, a nominal 10,000 patients are initially allocated to treatment and distributed between four sUA sub-states ( $<6,6$ to $<8,8$ to $<10$ and $\geq 10 \mathrm{mg} / \mathrm{dL}$ ) based on the results of PKPD simulations. In each model cycle, a proportion of patients discontinue treatment and are redistributed between the sUA sub-states to an untreated sUA distribution. A proportion also move to a dead state according to all-cause mortality probabilities derived from life tables for England and Wales in $2015 .{ }^{44}$ The model conservatively assumes that the only benefit of reducing sUA concentrations is to reduce the frequency of acute gout flares. A flare frequency distribution was derived from cross-sectional survey data ${ }^{45}$ across five categories; $0,1-2,3,4-5$ and $6+$ flares per annum. Fewer gout flares then result in improved quality of life and reduced treatment costs. ${ }^{45}$

\section{Adherence Data}

Adherence to ULTs was assumed from real world data on chronic treatment (119 subjects, 15,959 individual doses and follow-up between 90 and 529 days $\left.{ }^{46,47}\right)$ using electronicallyrecorded pill bottle cap opening times ${ }^{48}$ (MEMS, Aardex Group). Many of the adherence patterns are characterised by an implementation phase of varying levels of adherence followed by a complete cessation of doses prior to the end of the observation period. Instances of non-adherence following the implementation phase were discarded, as discontinuation was modelled separately in the pharmacoeconomic model. The distribution of dose implementation is given in Table S1, while figures showing all doses taken by every subject are provided in Figures S3-S7 in the Supplementary Material.

\section{Simulation Modelling}

Linked PKPDPE simulations were performed for each of the 14 ULTs. The pharmacometric stage was performed for each of the 119 real world adherence patterns ranging from 57 days to 529 days of dose implementation. Each simulation was repeated 500 times with 
resampling from individual random effects and from the probability distributions (based on clinical trial baseline data ${ }^{19}$ ) assumed for subject covariates including age (log-normal), weight (log-normal) and baseline sUA concentration (normal). However, uncertainty in the parameter estimates, in the estimates of the random effects parameters and residual variability was not included in PKPD simulations.

The simulated sUA time courses were used to generate post-treatment sUA concentration distributions across four states for use in the pharmacoeconomic model. These were obtained by taking the mean of the simulated daily sUA levels for days beyond day 50 but before discontinuation. The primary measure of treatment response for a ULT, equivalent to the primary outcome measure used in many clinical trials ${ }^{17-19}$, is the proportion of subject simulations in the $<6 \mathrm{mg} / \mathrm{dL}$ state. Further alternative measures of treatment response were derived using daily sUA concentrations for all available days beyond day 50 but before discontinuation. The proportions of subject simulations which were $<6 \mathrm{mg} / \mathrm{dL}$ for at least 80 , 70 , or $60 \%$ of days have been calculated to measure sustained response. Although not reported in clinical trials, there are more likely to be associated with a reduction in gout symptoms $^{49}$.

In each iteration of the pharmacoeconomic model, the process of collapsing sUA concentration measurements on to the four sUA states was repeated with random sampling to bootstrap and propagate PKPD variability. Other pharmacoeconomic model inputs, such as flare frequency distributions, health state utilities and discontinuation rates, were also varied according to probability distributions used to represent uncertainty regarding their true value. Further details of the model parameters and probability distributions are summarised in the Supplementary Material. A total of 5,000 pharmacoeconomic models were performed for each unique ULT and adherence pattern combination.

The outputs of the pharmacoeconomic model for each ULT are the mean per patient lifetime QALYs and costs associated with gout following the initiation of treatment and the mean number of cycles of drug supplied. All hypothetical ULTs were compared with febuxostat 80 
mg or $120 \mathrm{mg}$, both with an annual price of $£ 317.72 .{ }^{40}$ Cost effectiveness thresholds were used to determine whether a higher cost treatment is sufficiently effective to justify reimbursement. We have used a cost effectiveness threshold of $£ 20,000$ per QALY gained which is routinely used in the UK. ${ }^{50}$

Where a hypothetical ULT is more effective than febuxostat $80 \mathrm{mg}$ we have estimated the maximum price at which the hypothetical ULT would be cost effective using the mean QALY and cost differences. The maximum cost effective price can be found by solving equation 1 for $P_{h}$ when net monetary benefit (NMB) is equal to zero.

$N M B=\lambda \Delta Q-\left(\Delta C_{N D}+\left(S_{h} * P_{h}-S_{f} * P_{f}\right)\right)$

NMB is the net monetary benefit, $\lambda$ is the cost effectiveness threshold, $\Delta Q$ is the difference in lifetime QALYs, $\Delta C_{N D}$ is the difference in non-drug costs, $S_{f}$ is the number of cycles febuxostat $80 / 120 \mathrm{mg}, P_{f}$ is the price of febuxostat $80 / 120 \mathrm{mg}, S_{h}$ is the number of cycles of hypothetical ULT, and $P_{h}$ is the price of the hypothetical ULT.

\section{ACKNOWLEDGEMENTS}

We acknowledge the support of the Supercomputing Wales project, which is part-funded by the European Union's Convergence European Regional Development Fund, administered by the Welsh Government.

\section{AUTHOR CONTRIBUTIONS}

D.H.-M., E.S., S.M., and D.A.H. wrote the manuscript; D.H.-M. and D.A.H. designed the research; D.H.-M. performed the research; D.H.-M. analyzed the data. 


\section{STUDY HIGHLIGHTS}

\section{WHAT IS THE CURRENT KNOWLEDGE ON THE TOPIC?}

Linked pharmacometric and pharmacoeconomic modelling has been shown to have potential utility across a range of different applications.

\section{WHAT QUESTION DID THIS STUDY ADDRESS?}

This study has sought to extend linked pharmacometric and pharmacoeconomic models to account for realistic drug adherence and quantify the value of drugs with improved forgiveness to missed doses.

\section{WHAT DOES THIS STUDY ADD TO OUR KNOWLEDGE?}

This was a case study of the application of linked pharmacometric and pharmacoeconomic models to quantify the value of hypothetical xanthine oxidase inhibitors for treating gout, based on their pharmacology. The maximum reimbursement price for hypothetical agents with increased potency or reduced clearance was estimated based on treatment success rates. This novel framework provides a direct link between drug pharmacology and the probability of a drug being cost-effective while explicitly accounting for realistic medication adherence.

HOW MIGHT THIS CHANGE CLINICAL PHARMACOLOGY AND THERAPEUTICS? These methods have the potential to inform early drug development by providing an indication of whether drug candidates possess the properties that would result in a maximum reimbursement price that justifies their progression through the long and costly drug development process. 


\section{REFERENCES}

1. Hill-Mcmanus, D., Marshall, S., Soto, E., Lane, S. \& Hughes, D. A. Impact of nonadherence and flare resolution on the cost effectiveness of treatments for gout: Application of a linked pharmacometric/pharmacoeconomic model. Value Heal. 21, 1373-1381 (2018).

2. Slejko, J. F., Willke, R. J., Ribbing, J. \& Milligan, P. Translating Pharmacometrics to a Pharmacoeconomic Model of COPD. Value Heal. 19, 1-7 (2016).

3. Hoogendoorn, M. et al. Cost-effectiveness models for chronic obstructive pulmonary disease: cross-model comparison of hypothetical treatment scenarios. Value Heal. 17, 525-536 (2014).

4. Pink, J., Lane, S. \& Hughes, D. A. Mechanism-based approach to the economic evaluation of pharmaceuticals:

Pharmacokinetic/pharmacodynamic/pharmacoeconomic analysis of rituximab for follicular lymphoma. Pharmacoeconomics 30, 413-429 (2012).

5. Rubio, A., Cox, C. \& Weintraub, M. Prediction of diltiazem plasma concentration curves from limited measurements using compliance data. Clin. Pharmacokinet. 22, 238-246 (1992).

6. Hughes, D. A. Estimation of the impact of noncompliance on pharmacokinetics: an analysis of the influence of dosing regimens. Br. J. Clin. Pharmacol. 65, 871-878 (2008).

7. Hill-McManus, D., Soto, E., Marshall, S., Lane, S. \& Hughes Impact of non-adherence on the safety and efficacy of uric acid-lowering therapies in the treatment of gout. $\mathrm{Br}$. J. Clin. Pharmacol. 84, 142-152 (2018).

8. Vrijens, B., Claeys, M. J., Legrand, V., Vandendriessche, E. \& Werf, F. Van de Projected inhibition of platelet aggregation with ticagrelor twice daily vs. clopidogrel 
once daily based on patient adherence data (the TWICE project). Br. J. Clin. Pharmacol. 77, 746-755 (2014).

9. Challenger, J. D., Bruxvoort, K., Ghani, A. C. \& Okell, L. C. Assessing the impact of imperfect adherence to artemether-lumefantrine on malaria treatment outcomes using within-host modelling. Nat. Commun. 8, 1373 (2017).

10. Swift, B. et al. Innovation at the Intersection of Clinical Trials and Real-World Data Science to Advance Patient Care. Clin. Transl. Sci. 11, 450-460 (2018).

11. Blaschke, T. F., Osterberg, L., Vrijens, B. \& Urquhart, J. Adherence to Medications: Insights Arising from Studies on the Unreliable Link Between Prescribed and Actual Drug Dosing Histories. Annu. Rev. Pharmacol. Toxicol. 52, 275-301 (2012).

12. Vrijens, B. et al. A new taxonomy for describing and defining adherence to medications. Br. J. Clin. Pharmacol. 73, 691-705 (2012).

13. Osterberg, L. G., Urquhart, J. \& Blaschke, T. F. Understanding forgiveness: Minding and mining the gaps between pharmacokinetics and therapeutics. Clin. Pharmacol. Ther. 88, 457-459 (2010).

14. Assawasuwannakit, P., Braund, R. \& Duffull, S. B. A framework for quantifying the influence of adherence and dose individualization. Clin. Pharmacol. Ther. 99, 452459 (2016).

15. Stauffer, M. E., Hutson, P., Kaufman, A. S. \& Morrison, A. The Adherence Rate Threshold is Drug Specific. Drugs R D 17, 645-653 (2017).

16. Hughes, D. A. \& Walley, T. Predicting 'real world' effectiveness by integrating adherence with pharmacodynamic modeling. Clin. Pharmacol. Ther. 74, 1-8 (2003).

17. Becker, M. A. et al. Febuxostat Compared with Allopurinol in Patients with Hyperuricemia and Gout. N. Engl. J. Med. 353, 2450-2461 (2005). 
18. Schumacher, H. R. et al. Effects of febuxostat versus allopurinol and placebo in reducing serum urate in subjects with hyperuricemia and gout: A 28-week, phase III, randomized, double-blind, parallel-group trial. Arthritis Care Res. 59, 1540-1548 (2008).

19. Becker, M. A. et al. Clinical Efficacy and Safety of Successful Longterm Urate Lowering with Febuxostat or Allopurinol in Subjects with Gout Clinical Efficacy and Safety of Successful Longterm Urate Lowering with Febuxostat or Allopurinol in Subjects with Gout. J. Rheumatol. 36, 1273-1282 (2009).

20. Saag, K. G. et al. Lesinurad Combined With Allopurinol: Randomized, Double-Blind, Placebo-Controlled Study in Gout Subjects With Inadequate Response to Standard of Care Allopurinol (A US-based Study). Arthritis Rheumatol. 69, 203-212 (2017).

21. Bardin, T. et al. Lesinurad in combination with allopurinol: a randomised, double-blind, placebo-controlled study in patients with gout with inadequate response to standard of care (the multinational CLEAR 2 study). Ann. Rheum. Dis. 76, 811-820 (2016).

22. Khanna, P., Khanna, D., Storgard, C., Baumgartner, S. \& Morlock, R. A world of hurt: Failure to achieve treatment goals in patients with gout requires a paradigm shift. Postgrad. Med. 128, 34-40 (2016).

23. Cottrell, E., Crabtree, V., Edwards, J. J. \& Roddy, E. Improvement in the management of gout is vital and overdue: An audit from a UK primary care medical practice. BMC Fam. Pract. 14, 170 (2013).

24. Roddy, E., Packham, J., Obrenovic, K., Rivett, A. \& Ledingham, J. M. Management of gout by UK rheumatologists: a British Society for Rheumatology national audit. Rheumatology (Oxford). 57, 826-830 (2018).

25. Yin, R. et al. Rate of adherence to urate-lowering therapy among patients with gout: a systematic review and meta-analysis. BMJ Open 8, e017542 (2018). 
26. Scheepers, L. E. J. M. et al. Medication adherence among patients with gout: A systematic review and meta-analysis. Semin. Arthritis Rheum. 47, 689-702 (2018).

27. Šmelcerović, A. et al. Xanthine oxidase inhibitors beyond allopurinol and febuxostat; an overview and selection of potential leads based on in silico calculated physicochemical properties, predicted pharmacokinetics and toxicity. Eur. J. Med. Chem. 135, 491-516 (2017).

28. Assawasuwannakit, P., Braund, R. \& Duffull, S. B. Quantification of the Forgiveness of Drugs to Imperfect Adherence. CPT Pharmacometrics Syst. Pharmacol. 4, 204$211(2015)$

29. Sanchez-Nino, M. D. et al. Lesinurad: What the nephrologist should know. Clin. Kidney J. 10, 679-687 (2017).

30. National Institute for Health and Care Excellence Final appraisal determination: Pegloticase for treating severe debilitating chronic tophaceous gout. (2013). <https://www.nice.org.uk/guidance/ta291>

31. Hasselt, J. G. C. van et al. Integrated Simulation Framework for Toxicity, Dose Intensity, Disease Progression, and Cost Effectiveness for Castration-Resistant Prostate Cancer Treatment With Eribulin. CPT Pharmacometrics Syst. Pharmacol. 4, 374-385 (2015).

32. Pink, J., Pirmohamed, M., Lane, S. \& Hughes, D. A. Cost-effectiveness of pharmacogenetics-guided warfarin therapy vs. alternative anticoagulation in atrial fibrillation. Clin. Pharmacol. Ther. 95, 199-207 (2014).

33. Kamal, M. A. et al. Interdisciplinary pharmacometrics linking oseltamivir pharmacology, influenza epidemiology and health economics to inform antiviral use in pandemics. Br. J. Clin. Pharmacol. 83, 1580-1594 (2017).

34. Vrijens, B. et al. Successful Projection of the Time Course of Drug Concentration in 
Plasma During a 1-Year Period From Electronically Compiled Dosing- Parameterized Pharmacokinetic Models. J. Clin. Pharmacol. 45, 461-467 (2005).

35. Milligan, P. A. et al. Model-based drug development: a rational approach to efficiently accelerate drug development. Clin. Pharmacol. Ther. 93, 502-14 (2013).

36. Beard, S. M., Scheele, B. G. Von, Nuki, G. \& Pearson, I. V Cost-effectiveness of febuxostat in chronic gout. Eur. J. Heal. Econ. 15, 453-463 (2014).

37. Perez-Ruiz, F., Diaz-torne, C. \& Carcedo, D. Cost-effectiveness analysis of febuxostat in patients with gout in Spain. J. Med. Econ. 19, 604-610 (2016).

38. National Institute for Health and Care Excellence Single Technology Appraisal [ID761]: Lesinurad for treating chronic hyperuricaemia in people with gout, Committee Papers. (2016).

39. Li, X. et al. Serum uric acid levels and multiple health outcomes: umbrella review of evidence from observational studies, randomised controlled trials, and Mendelian randomisation studies. BMJ 357, j2376 (2017).

40. Joint Formulary Committee British National Formulary (online) London: BMJ Group and Pharmaceutical Press. (2018). <bnf.nice.org.uk>

41. National Institute for Health and Care Excellence. Guide to the single technology appraisal process. (2009). <https://www.nice.org.uk>

42. National Institute for Health and Care Excellence Guide to the methods of technology appraisal 2013. (2013). <nice.org.uk/process/pmg9>

43. R Core Team R: A language and environment for statistical computing. R Foundation for Statistical Computing. (2017). <https://www.r-project.org/>

44. The Office for National Statistics England and Wales 2015. Natl. Life Tables (2015). $<$ https://www.ons.gov.uk/peoplepopulationandcommunity/birthsdeathsandmarriages/lif 
eexpectancies>

45. Khanna, P. P. et al. Tophi and frequent gout flares are associated with impairments to quality of life, productivity, and increased healthcare resource use: Results from a cross-sectional survey. Health Qual. Life Outcomes 10, 117 (2012).

46. Vrijens, B. \& Goetghebeur, E. The impact of compliance in pharmacokinetic studies. Stat. Methods Med. Res. 8, 247-262 (1999).

47. Bovet, P. et al. One-year compliance to medication in newly diagnosed hypertensive patients in a developing country. Can. J. Cardiol. 13, (1997).

48. Urquhart, J. The electronic medication event monitor. Lessons for pharmacotherapy. Clin. Pharmacokinet. 32, 345-356 (1997).

49. Shoji, A., Yamanaka, H. \& Kamatani, N. A Retrospective Study of the Relationship Between Serum Urate Level and Recurrent Attacks of Gouty Arthritis : Evidence for Reduction of Recurrent Gouty Arthritis With. Arthritis Rheum. (Arthritis Care Res.) 51, $321-325(2004)$

50. McCabe, C., Claxton, K. \& Culyer, A. J. The NICE cost-effectiveness threshold: what it is and what that means. Pharmacoeconomics 26, 733-744 (2008). 

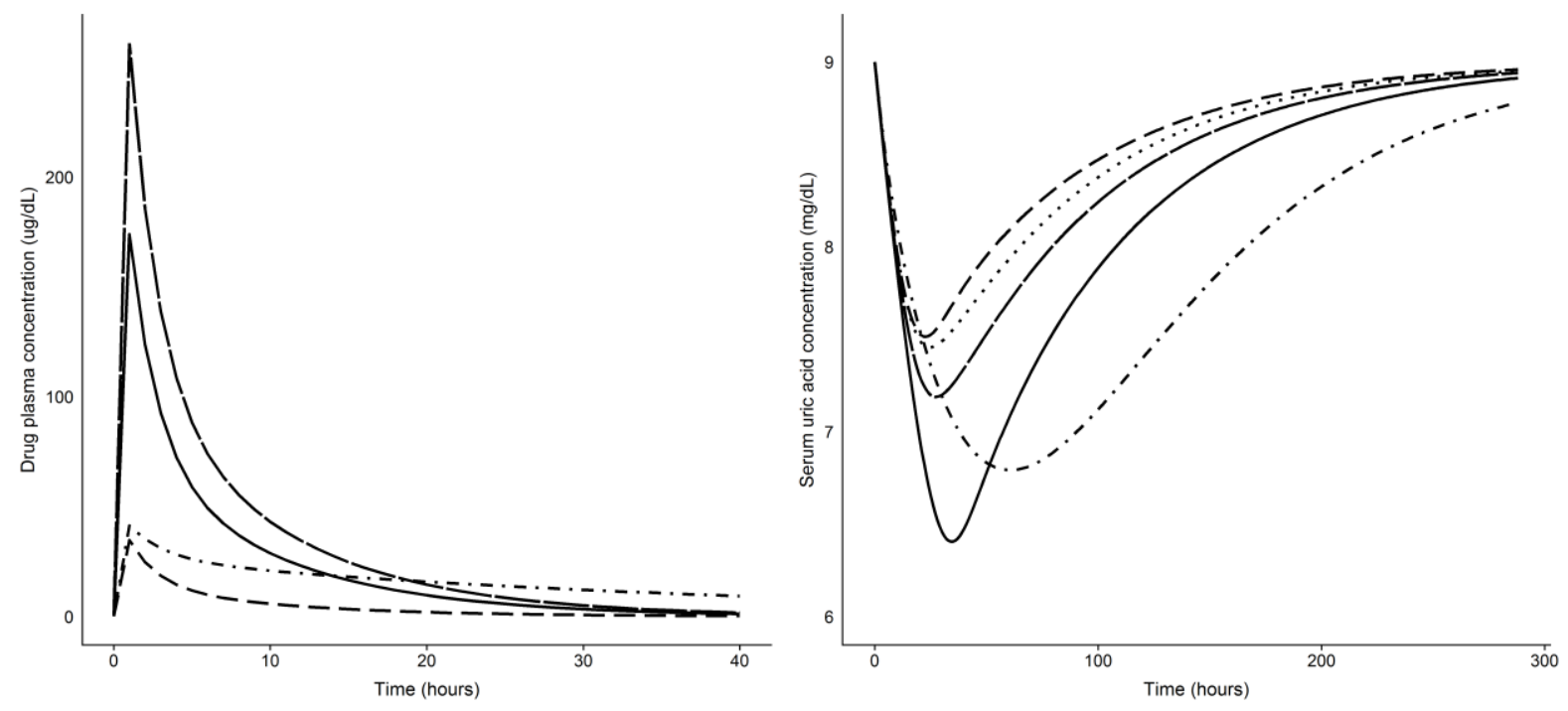

ULT simulation

A4/FBX80

$\begin{array}{lll}-- & B 4 \\ -\cdots & C 4\end{array}$

- FBX120

ULT simulation

$\begin{array}{lll} & \text { A4 } \\ -- & \text { B4 } \\ - & & \text { C4 }\end{array}$

- $\mathrm{FBX} 120$

…. FBX80

Figure 1. Simulated drug plasma concentration and serum uric acid time course following a single oral dose (taken at hour 12) of febuxostat $80 \mathrm{mg}$ or $120 \mathrm{mg}$ as well as 3 hypothetical ULTs (simulations used a reference subject of age 60, weight $100 \mathrm{~kg}$, and baseline sUA of 9 $\mathrm{mg} / \mathrm{dL})$ 


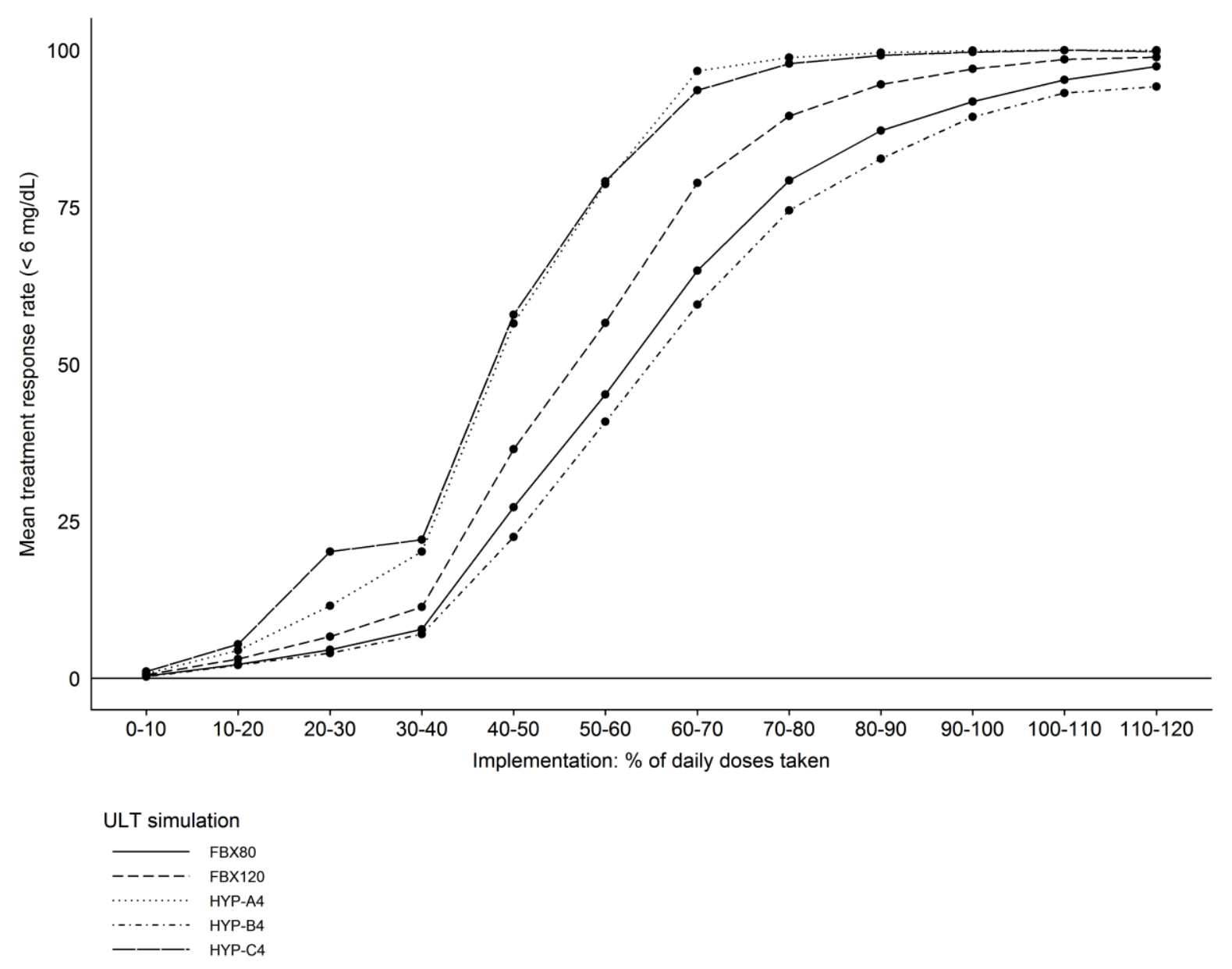

Figure 2. Mean treatment response rates for hypothetical ULTs compared with febuxostat $80 \mathrm{mg}$ by dose implementation using response defined using mean daily sUA concentration below $6 \mathrm{mg} / \mathrm{dL}$ target 


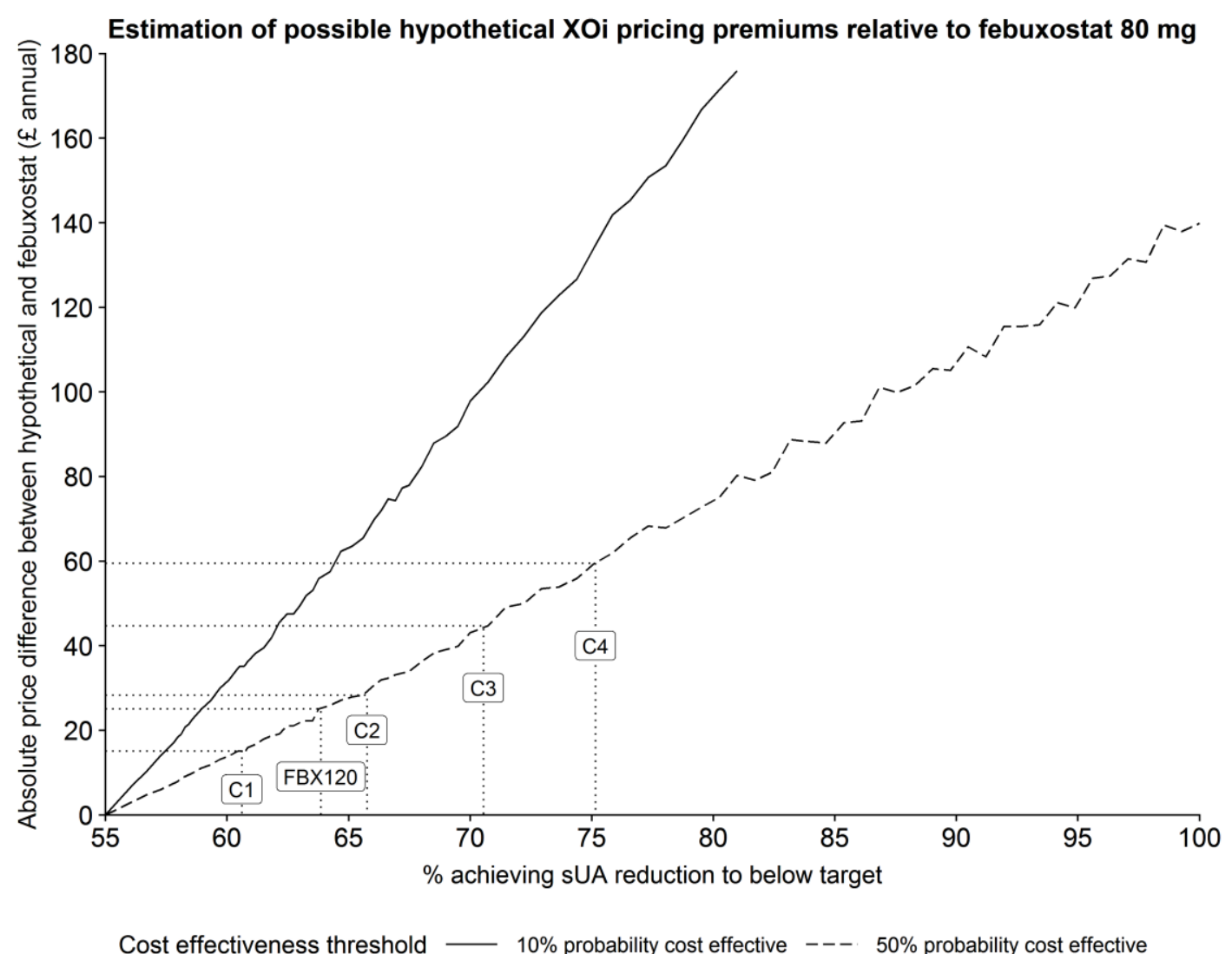

Figure 3. Curve of estimated pricing to achieve cost effectiveness versus febuxostat $80 \mathrm{mg}$ with probability of $50 \%$ and $10 \%$ at a willingness to pay threshold of $£ 20,000$ 


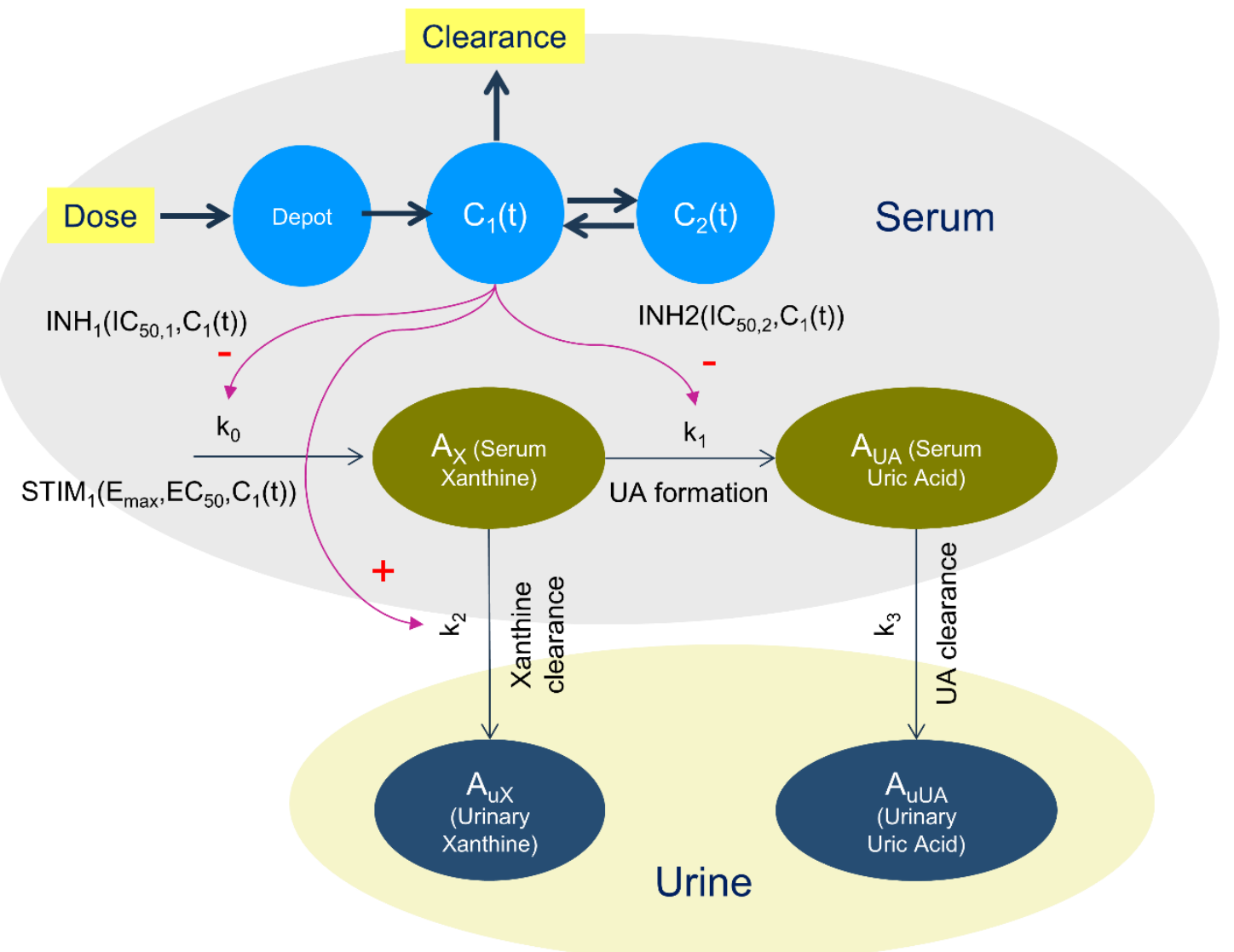

Figure 4. Diagrammatic and mathematical representations of the pharmacodynamics of dual-urate lowering therapies. $\mathrm{k}_{0}, \mathrm{k}_{1}, \mathrm{k}_{2}$ and $\mathrm{k}_{3}$ are the rate parameters for the production of xanthine, xanthine to uric acid conversion, removal of xanthine to urine and removal of uric acid to urine, respectively. INH functions. Ax and $A_{\cup A}$ are the total time-varying amounts of xanthine and uric acid in serum, respectively. $A_{u x}$ and $A_{u \cup A}$ are total amounts of xanthine and uric acid removed to urine, respectively. $\mathrm{C}_{1}(\mathrm{t})$ and $\mathrm{C}_{2}(\mathrm{t})$ are the plasma concentrations of drug in the central and peripheral pharmacokinetic compartments respectively. 